Ewa Stala (D)

Uniwersytet Jagielloński ewa.stala@uj.edu.pl

\title{
Historia de la lexicografía hispanoamericana
}

\section{Resumen:}

El presente texto es una aproximación a la historia de la lexicografía hispanoamericana -área de la lexicografía española relativamente poco mencionada en los estudios lexicográficos generales. Siendo este tema bastante amplio, empezamos con la lexicografía hispano-amerindia en la etapa colonial: desde las primeras obras hasta la etapa de independencia. A continuación presentamos los principales logros de la lexicografía hispanoamericana contemporánea para luego dedicar un párrafo a los retos de la metalexicografía en la América Hispanohablante. Para una mejor comprensión, precedemos el texto principal con un breve esbozo histórico-cultural.

Palabras clave: historia de la lexicografía española, lexicografía hispano-amerindia, lexicografía mono-, bi- y multilingüe, metalexicografía

\footnotetext{
Abstract:

The History of Hispanic-American Lexicography

The present text is a summary of the history of Hispanic-American lexicography - an area of Spanish lexicography that is relatively rarely discussed in general lexicographic studies. As this topic is quite broad, the article opens with a discussion of the Hispanic-Amerindian lexicography in the colonial period from the first works to the independence stage. Then the main achievements of contemporary Hispanic-American lexicography are presented. At the end, a paragraph is dedicated to the challenges of the metalexicography in Spanish-speaking America. For a better understanding, the main text is preceded with a brief historical-cultural outline.
} 
Keywords: history of Spanish lexicography, Hispanic-Amerindian lexicography, mono-, bi- and multilingual lexicography, metalexicography

\section{Introducción}

Si bien existen numerosos estudios sobre la llamada lingüística misionera ${ }^{1} \mathrm{y}$ hace poco apareció una monografía dedicada exclusivamente a los diccionarios hispano-amerindios ${ }^{2}$, en los manuales y trabajos generales sobre la lexicografía del español la parte hispanoamericana no suele ocupar mucho espacio ${ }^{3}$ y se tiene en menor consideración las fuentes primarias relativas a este tema. Hecho que puede extrañar si tenemos en cuenta que los primeros repertorios americanos impresos preceden a los diccionarios de español y lenguas europeas tales como italiano, inglés o francés. De hecho, hace unas décadas Gili Gaya (1947) dijo de estos diccionarios que "nada añaden a los europeos" (aunque, hay que admitirlo, Menéndez Pidal (1944) planteó la importancia de este tipo de investigaciones). Además, el término mismo lexicografía hispanoamericana es muy amplio: puede concernir a la lexicografía bilingüe hispano-amerindia, las obras lexicográficas publicadas en América, pero también la lexicografía "regional" o cuestiones metalexicográficas.

Dada la amplitud del tema, en este trabajo nos enfocamos en la historia de la lexicografía de las lenguas indígenas y el español para luego ampliar el panorama, aunque sea parcialmente, con las obras

1 Nos referimos sobre todo a la serie dirigida por Otto Zwartjes, Missionary Linguistics/ Lingüística Misionera (2004-2014), los trabajos publicados por la editorial Iberoamericana con el mismo nombre, así como la colección El Paraíso en el Nuevo Mundo.

2 Hernández (2018).

3 Por ejemplo, en la obra de Medina Guerra (2003) de 427 páginas, este tema ocupa las páginas 186-189. En la obra de Azorín Fernández (2004) ni siquiera aparece, tal y como ocurre, por ejemplo, en el caso de la monografía de Alvar Ezquerra (2002). 
más recientes y algunos aspectos metalexicográfícos. Para ubicar toda esta producción en un contexto, empezamos con un pequeño esbozo histórico- cultural.

\section{Esbozo histórico-lexicográfico-cultural}

En 1490 Alfonso de Palencia publica en Sevilla su Uniuersale Compendium Vocabulorum (Vocabulario universal en latín y en romance), un diccionario bilingüe que resulta muy útil para el estudio de la lengua romance y representa el primer esfuerzo lexicográfico en la lengua castellana. Dos años más tarde E. A. de Nebrija publica su Gramática de la lengua castellana y Diccionario latino-español. El mismo Nebrija unos años más tarde (1495) publica su Vocabulario español-latino. Como bien se sabe, en la misma época Colón llega a la "pequeña España", o sea la Hispaniola, abriendo una nueva etapa en la historia mundial.

Margarita Hidalgo (en: Conde Silvestre, 2007: 227) presenta el siguiente resumen de las etapas de la colonización:

1. años 1521-1565: cuando es obligatorio aceptar el credo romano y en el nivel de la lengua no hay mucho interés por el bilingüismo, lo que en el nivel de la política lingüística equivaldrá a imposición castellanista y, por consiguiente, uniformización,

2. época de Felipe II (1527-1598, rey de España entre 1556-1598): cuando aumenta el interés por las lenguas amerindias; en $1580 \mathrm{Fe}-$ lipe II dispuso establecer cátedras de lenguas indígenas y en 1583 el tercer Concilio Limense decide que los sacerdotes que quieran emprender la misión evangelizadora deben dominarlas,

3. siglo XVIII: cuando, por orden de Carlos III, se relegan las lenguas autóctonas del espacio evangelizador, en 1770 se expulsan los jesuitas de España y América y se produce la imposición del español, y subraya que el lugar del contacto lingüístico lo constituyeron sobre todo las ciudades, mientras que en las áreas rurales ese contacto fue mínimo. A pesar de ello, veremos que esta primera etapa también se deja conocer como el momento de aparición de algunas obras lexicográficas. 
Durante los primeros siglos de la presencia hispana en el continente americano hubo, de hecho, dos centros de imprenta y mercados de libros: México y Lima, sedes de los primeros dos virreinatos establecidos por los españoles ${ }^{4}$. Es en la ciudad de México, donde, en la esquina de las calles Moneda y Licenciado Primo Verdad, Gerónimo de Aguilar en 1524 funda la primera imprenta en el Nuevo Mundo. La segunda, en Lima, aparecerá 40 años más tarde.

Como bien observa Acero Durántez (2003: 186), la lexicografía amerindia en la época colonial tuvo como objetivo paliar las necesidades comunicativas tanto por parte de los misioneros (principalmente dominicos, franciscanos y jesuitas) como por los administradores coloniales ya que "además de expandir el mensaje del evangelio, había que tratar con la fuerza laboral indígena". Los misioneros, forzados a aprender lenguas tipológicamente tan diversas, encontraron una dificultad adicional: la multiplicidad de lenguas y dialectos. A modo de ejemplo: en lo que hoy es México había 125 lenguas diferentes (Acero Durántez, 2003: 186) y en el territorio argentino, 35 (Censabella, 1999: 17). El cambio del área de la evangelización suponía la necesidad de cambiar el idioma, así algunos misioneros predicaban en dos o tres lenguas diferentes o más, como el famoso Andrés de Olmos que conocía hasta diez ${ }^{5}$. A mediados del siglo XVI empiezan a describirse las lenguas amerindias y aparecen sus gramáticas, pero también empieza a inventariarse el léxico. Para ello se necesita un modelo previo. Según Gili Gaya (1947), el vocabulario español-latino de Nebrija constituyó la primera fuente de los vocabularios bilingües con las entradas en español. Acero Durántez (2003) menciona a Calepino y Nebrija, Martínez (1968) menciona a Nebrija y Palencia. Se aplican también nuevos métodos; además en muchos casos al principio se agregan informaciones acerca de la fonética, la gramática y el léxico

4 Martínez (1968) cita también la zona del Caribe con su Diccionario caribefrancès (1665) de Breton, pero por no contener esta obra español, la dejamos de lado. Para más detalles sobre la lectura en los tiempos coloniales recomendamos la obra de García Aguilar y Rueda Ramírez (compiladores) del año 2010 o la de Guibovich Pérez (2019).

5 Para la biografía de este franciscano, véase Zaldívar, en línea. 
de las lenguas amerindias, pero también se dan casos de falta de equivalencia, descripción en vez de un simple equivalente o un visible cruce de sendas lenguas. El producto principal es un diccionario bilingüe, pero hay otros productos como glosarios en los que se documentan las glosas indígenas o manuscritos; son ejemplo de ello los de lenguas mayas que tienen como lengua de partida la indígena. Los autores, casi todos misioneros, fueron los que crearon convenciones gráficas, codificación gramatical, compilación léxica. En su labor resultaron decisivos los dos primeros siglos, cuando no solo compilaron el léxico, sino también agregaron informaciones acerca de la gramática de las lenguas indígenas. Como acertadamente observa Martínez (1968), la diferencia entre los diccionarios publicados en España y América yace, entre otras, en que mientras los primeros (i.e. el de Palencia o Nebrija) se publican cuando la lengua está en la plenitud de su unificación, en América llega a un territorio vasto, remoto y diverso. Esta diferencia es visible al menos de una manera: en América el estudio del inventario léxico precede a la consideración de la lengua desde el punto de vista de la gramática, hecho parcialmente debido también a la falta de lenguas clásicas.

\section{Historia de la lexicografía bi- y multilingüe de la etapa colonial}

No hace tanto López Morales (2000: 61) advertía: "la historiografía lingüística hispanoamericana es de una debilidad extrema, especialmente en cuanto a diccionarios se refiere". Y, tal y como se ha mencionado, en 2018 se publicó un trabajo elaborado por Esther Hernández: un compendio de carácter enciclopédico dedicado a la lexicografía colonial hispano-amerindia en el periodo 1550-1800 o sea desde los principios hasta la etapa de independencias. Existen algunos trabajos previos: sobre los vocabularios mayas, confeccionados por los franciscanos (Bolles, 2003), la monografía de Karttunen (1995) sobre la lexicografía mesoamericana, los trabajos de Hans-Joseph Niederehe (2004-2015), la citada serie de Otto Zwartjes. También, como menciona la misma Hernández, una descripción general se encuentra en 
el Wörterbücher. Dictionaries. Dictionnairies: ein internationales Handbuch zur Lexicographie, editada por Hausmann et al. en 1991. La bibliografía de Fabbri (1979) en las páginas 277-311 presenta los diccionarios americanos dividiéndolos en bilingües (pero solo abarca lenguas tales como: inglés, esperanto, alemán, italiano, ruso, etc.), políglotas y luego las obras regionales, pero no es un recuento completo. Obviamente hay que citar, aunque solo sea como punto de referencia, la bibliografía del Conde de la Viñaza (1892): Bibliografía española de lenguas indígenas de América o el más reciente Diccionario bibliográfico de la metalexicografía del español, dirigido por Ignacio Ahumada (2006-2014).

Los orígenes de la lexicografía amerindia fundamentalmente empiezan con las glosas, intercaladas en los textos impresos, por ejemplo en el Vocabulario de Molina (1555), pero también con relatos de viaje o hasta poemas ${ }^{6}$. Aunque el primer testimonio lexicográfico es un vocabulario de verbos nahuas atribuido al mencionado fray Andrés de Olmos, probablemente del año 1547, es más una lista de palabras que un glosario propiamente dicho. Su ausencia en los manuales tal vez se deba a que es un manuscrito y las obras impresas son más famosas y más fáciles de describir, mientras que los manuscritos son más dificultosos en cuanto a la fecha, autoría, copia y paradero, muchas veces desconocido ${ }^{7}$. El vocabulario de Olmos se encuentra agregado a su Arte de la lengua mexicana, la primera gramática de una lengua indígena redactada en el Nuevo Mundo, y es una lista de verbos nahuas con las correspondencias en castellano más una lista con las entradas en castellano. Toda la obra cuenta con 288 folios y la lista de verbos abarca 63 folios (Hernández, 2018: 55). Olmos se inspiró en las Introductiones latinae de Nebrija (c.1488) y lo menciona en su

6 Nos referimos a las autoglosas de los vocablos americanos que Martín del Barco Centenera inserta en su poema Argentina y conquista del Río de la Plata (1602); para más detalles sobre este tipo de glosas véase Barcia (2004: 25-42).

De hecho, la obra fue publicada como facsímil en la Universidad Autónoma de México, Instituto de Investigaciones Históricas, en 2002. Para 2020 (con la fecha de 2019) se espera la publicación de la primera edición crítica con estudio preliminar, edición y notas de Heréndira Téllez Nieto. 
prólogo. En las definiciones y las entradas encontramos traducciones literales del náhuatl al castellano como: "afeitarse la muger a su modo antiguo" o "verde cosa no madura". Fue el texto básico usado por los misioneros en la codificación gramatical de las lenguas amerindias, sirvió de fuente para las publicaciones posteriores ( $c f$. Molina, 1555 y 1571) y es una prueba de la existencia de la escuela lexicográfica franciscana ${ }^{8}$.

El primer glosario propiamente dicho es una obra anónima de mitad del s. XVI, titulada Vocabulario trilingüe castellano, latino y mexicano. Si bien se discute tanto su autoría (fray Bernardino de Sahagún/ un hablante nativo de náhuat1/ un misionero en colaboración con los indígenas), como su fecha (c. 1550/ 1540-1555/ antes de 1555/ 15401570), lugar (colegio de Tlatelolco/ otro colegio de este tipo) y hasta objetivo (un diccionario pasivo de latín y español para los hablantes nahuas/ un diccionario de náhuatl para los evangelizadores católicos) ${ }^{9}$. Casi todos admiten que, como en el caso de la mayoría de los diccionarios del Nuevo Mundo, la fuente principal del Vocabulario trilingüe fue el diccionario español-latino de Nebrija, aunque no la primera versión de c. 1495, sino la de 1516 o una versión pirata de 1520 (Gruda, 2018). El manuscrito, bien encuadernado, contiene 157 hojas de papel europeo (probablemente de Génova) en formato 18x13.5 (en España denominado cuarto menor), lleva el título en el lomo, posee dos marcas de agua y en su encabezamiento figura como título: Dictionarium ex hisniensi [sic] in latinum sermonem interprete Aelio Antonio $\mathrm{Ne}$ prissensi. Lege foeliciter. La encuadernación es posterior, así como la numeración de páginas.

Hernández (2018) lo describe como una copia del diccionario de Nebrija con las glosas manuscritas en náhuatl con tinta roja a doble columna. Las entradas son lexemas en español desde $A$ hasta zorzal, seguidas de sus equivalentes en latín y náhuatl. Los espacios blancos en la parte nahua sugieren que primero se realizó la copia del vocabulario

8 Sobre lo innovador de la versión de 1571, entre otros, véase Romero Angel (2006).

9 Más detalles sobre esta obra en Gruda (2018) o Hernández (2018: 56-60). 
de Nebrija y luego, donde resultó posible al autor, se agregaron las equivalencias indígenas. Aunque Hernández (2018: 54) lo describe como "una copia manuscrita del vocabulario español-latino de Nebrija, que lleva incorporadas glosas nahuas a todo el lemario", Gruda (2018) calcula que de unas 15260 entradas en español y latín, solo 10000 , o sea el $70 \%$, tienen su equivalente en náhuatl.

Como otras obras de esta índole, el vocabulario en cuestión es una muestra clara de cómo se correlacionaron las dos culturas y las dos lenguas. De hecho, el trabajo de Gruda está dedicado a los fenómenos del contacto cultural y contacto lingüístico entre las dos lenguas (castellano y náhuatl) y, a modo de ejemplo, enumeramos tras él algunos efectos de dicho contacto, a saber:

- préstamos CAMIXAHTLI < esp. CAMISA,

- calcos semánticos: ILHUICATL empieza a significar 'cielo' como el esp. cielo 'íd.' aunque el náhuatl diferencia las acepciones equivalentes como en inglés sky y heaven,

- traducciones literales: NECHIPAHUĀLŌYĀN 'lugar de limpieza' a semejanza del esp. PURGATORIO 'lugar de la purga, de limpieza',

- cambios semánticos: MAZĀTL ‘ciervo’ $\rightarrow$ ‘caballo’, TŌTŌL ‘pájaro' $\rightarrow$ 'pollo',

- híbridos: CAXTILLĀN CHICHÍTO lit. 'perro castellano', es decir 'blanchete', CAXTILLĀN MAZĀTL lit. 'ciervo castellano' o sea 'mula', 'oveja' o 'ganado'.

Gruda calcula que en el Vocabulario se documentan 245 entradas con los préstamos en español. Todos, tal como era de esperar, son sustantivos: aaron, ángel, ánima, asno, breviario, caballo, camisa, campana, candela, canón, capa, caso, Castilla, cristiano, cruz, cuchillo, Dios, emperador, greco, hora, hostia, januario, Jesucristo, judío, jueves, latín, lechuga, letra, Lucía, lunes, madre, marqués, miércoles, misal, obispo, october, padre, peso, pistacio, prouincia, queso, rábano, rocino, Roma, sabbato, sacramento, san(c)to, tocino, tono, viernes, vino, visorei, vocal. Llaman la atención las formas latinizantes: greco 'griego', hostia 'íd.', januario 'enero', sabbato 'sábado', visorei 'virrey' (ant. vicerrey). Si bien la forma hostia permanece hasta hoy 
día como latinismo en español, los demás vocablos han cambiado de forma a lo largo del tiempo (las formas actuales son: griego, enero, sábado, virrey). Curiosamente, estas voces no se documentan en el diccionario español-latino de Nebrija del año 1516. La consulta directa de la obra de Palencia ${ }^{10}$ muestra que en ella se hallan los siguientes lexemas: greco, hostia, ianuario y sabbato. La voz visorei o sus variantes (visorey, visorrey, etc.) no forma parte del repertorio de Palencia aunque sí se deja observar en varias obras de la época colonial (p. ej. Crónicas de los Reyes Católicos de Alonso de Santa Cruz 14911516, Cancionero de Antón de Montoro 1445-1480) o en numerosos documentos notariales de finales del siglo XV. Por todo ello, es de suponer que el autor no solo accedió al Vocabulario de Nebrija, sino tal vez se sirvió de la obra que quedó relegada al segundo plano: el Universal Vocabulario de Alfonso de Palencia (Baran, Stala, 2019).

Entre los primeros manuscritos se halla también el anónimo Glosario otomí (h. 1560) que contiene glosas otomíes agregadas al ejemplar del diccionario español-náhuatl de Molina (1555), escritas después de las equivalencias nahuas si hay espacio y, si no, en el interlineado o en los márgenes. Se atribuyen a fray Alonso Urbano, un predicador en otomí y náhuatl y se supone que es la primera codificación léxica de esta lengua. Otros glosarios manuscritos de esta época son: Glosario matlatzinca (anónimo, también al ejemplar de Molina 1555) y otros dos glosarios anónimos otomíes de 1559 y 1571.

De esta misma época, entre los manuscritos de autoría o datación incierta vale la pena mencionar Vocabulario de la lengua cakchiquel con advertencia de los vocablos de las lenguas quiché y zutujil (...) atribuido a fray Domingo de Vico, datado antes de 1555 ya que el dominico murió en ese año, donde la información léxica va intercalada por la información gramatical o Bocabulario en lengua Tzeldal (vocabulario tzeldal-hispano) atribuido a fray Domingo de Ara, c. 1560, el primer poblador de Copanaguastla (hoy quedan solo ruinas del

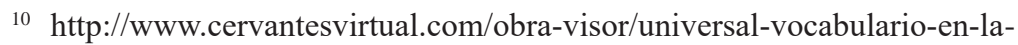
tin-y-en-romance-tomo-ii--0/html/003fa87a-82b2-11df-acc7-002185ce6064_509. html, 3.12.2020. 
convento abandonado del s. XVII). Contiene el vocabulario tzendalespañol, plegarias, nombres de pueblos traducidos en tzendal y oraciones. Su autor sigue el esquema nebrisense: coloca los lexemas casi sin información gramatical. La obra contiene muchas innovaciones léxicas en la parte castellana, lo que puede adelantar la fecha de documentación de algunos americanismos (Hernández, 2018: 66). Otro, llamado Vocabulario de Mayathan o Vocabulario de Viena es tal vez el primer diccionario castellano-maya yucateco (14 000 acepciones castellanas y 40000 acepciones mayas), tal vez es una copia de un manuscrito de la segunda mitad del s. XVI (o, según algunos, s. XVII). Discutible es no solo la fecha, sino también el lugar (convento San Miguel Arcángel en Maní? $)^{11}$ y el autor (tal vez un franciscano culto en colaboración con todo un equipo). La planta del diccionario está basada en el modelo nebrisense pero, curiosamente, no sigue el modelo de Molina, tan popular entre los francisanos y, cosa singular, contiene refranes (A buena hambre no ay pan malo, A moro muerto, gran lançada). Varias entradas están adaptadas a la realidad yucateca:

- cenote: arrojarse en el pozo o dsonote,

- curiosamente, contiene el quechuismo patata (Arrancar patatas y jícamas tirando de ellas o como se saca al asilado en iglesia) y también interesantes son los mayismos en el texto castellano del diccionario:

- Bolines, pescadillos pequeños: bolim.// De agua dulçe: topom,

- Cauar con los palos llamados xul, de puntas agudas: xul pan (Hernández, 2018: 73).

Un ejemplo más de la presencia de la tradición lexicográfica europea es el Vocabulario de la lengua maya de datación incierta (segunda mitad del s. XVI o principios del s. XVII). La primera parte, diccionario maya-español, suele denominarse Diccionario de Motul I porque tiene referencias a esta población; en 1984, al editarlo, se le dio el nombre de Calepino maya de Motul, y es la obra léxica maya

11 Uno de los conventos más relevantes de Yucatán, construido hacia 1559, obra del arquitecto Fray Juan Mérida, sede de los franciscanos (la orden Franciscana Menor cuyo escudo se halla a la entrada). 
más antigua, más completa y más importante de todas las conservadas y producidas en Yucatán durante los siglos XVI-XVII. La segunda parte, castellano-maya, todavía sin editar, suele denominarse Diccionario de Motul II. El autor de la parte mayo-hispana es probablemente fray Antonio de Ciudad Real y el de la parte hispano-maya es fray Alonso de Solana, ambos franciscanos. La segunda parte está basada en el modelo de Nebrija, pero también en las obras de Molina (1555, 1571) que estaban accesibles en los conventos franciscanos. En el nivel léxico destacan 45 indigenismos integrados en el texto castellano. Son sobre todo voces antillanas y nahuatlismos: batea, Mayz, tamales, Milpa, Milpero, papaya, Yguana, Mamey, Chocolate, xicara, naguas, Tomates, etc.

Entre las obras con paradero desconocido se notan sobre todo los vocabularios de lenguas de México y Guatemala: náhuatl, huasteco, tarasco, otomí, lengua popoloca (sic), maya, cakchiquel, quiché, tzutujil, zapoteca, pero también Arte y Vocabulario de la Lengua del Inca por Fr. Martin de Victoria, del Orden de la Merced, el primero que redujo á reglas dicho idioma (fecha desconocida) que figura en la bibliografía de Viñaza y que sería el primer vocabulario del quechua, Vocabulario de la lengua de los maramomis, una tribu brasileña, atribuido al jesuita Manuel Viegas, Diccionario de la lengua indígena del Brasil o sea tupí por otro jesuita P. José de Anchieta (c. 1590) y, finalmente, existe una copia del siglo XIX atribuida al jesuita Alonso de Bárcena: Lexica et praecepta grammatica, item liber confessionis et precum in quinque Indorum Linguis, quatan usus per American australem, nempre piquinica, teconotica, catamareana, guaranica, naitxaca, sive mogaznana (mogana), Peruviae 1590 y de acuerdo con la finalidad -pastoral- de este tipo de trabajos misioneros, va acompañada de un confesionario (conjunto de fórmulas que debían emplearse a la hora de impartir el sacramento de la confesión) y un libro de preces o devocionario. El Arte y vocabulario de la lengua toba, también de Bárcena, sería el primer diccionario de esta lengua, publicado en 1893 utilizando el manuscrito que se conserva en la Biblioteca Bartolomé Mitre. Se sabe que el jesuita en cuestión tuvo conocimiento práctico de once 
lenguas indígenas y escribió gramáticas, vocabularios y catecismos en gran número de ellas.

El diccionario de Alonso de Molina, Vocabulario de la lengua castellana y mexicana, es en realidad el primer diccionario impreso en el Nuevo Mundo. El autor, un franciscano bilingüe (emigró a América siendo niño), tuvo fama de ser el que mejor hablaba náhuatl y, de hecho, redactó varias obras para ayudar a sus compañeros. ${ }^{12} \mathrm{La}$ primera edición, de 1555, contiene más de 13000 voces, la segunda, de 1571, fue ampliada en 4000 términos castellanos y 22600 entradas nahuas y su título es: Vocabulario de la lengua castellana y mexicana, y mexicana y castellana (Acero Durántez, 2003: 187). Molina redactó ambas ediciones sirviéndose del Vocabulario español-latino de Nebrija, pero de su segunda edición del año 1516. Seguro acudió a otras fuentes (entre ellas la lista de verbos nahuas de fray Andrés de Olmos) y también consultó a otros hablantes bilingües. Los estudios manifiestan también una contribución notable del autor: el modelo nebrisense lo adapta a las necesidades de la lengua meta que es el náhuatl (p. ej. suprime voces sin equivalencia alguna), el orden es alfabético (y no etimológico o el de familias de palabras) más la advertencia de que el diccionario desde el principio fue pensado como un diccionario bilingüe bidireccional (lo admite en el Prólogo). Por todo ello, sigue siendo una obra de referencia para el náhuatl clásico ${ }^{13}$.

En 1559 se publica Vocabulario en lengua de Mechuacan (de la lengua tarasca y castellana, y castellana y tarasca) de Maturino Gilberti, otro franciscano de origen francés y ascendencia italiana ${ }^{14}$. Es el primer diccionario de la lengua tarasca, llamada también purépecha o michoacana, ya que el autor fue misionero de la lengua tarasca y dejó varias obras respecto a esta lengua ${ }^{15}$. Contiene 5000 lemas

${ }^{12}$ La bibliografía acerca de su vida y su obra es presentada por Hernández (2018: 94).

13 Sobre la gramática de náhuatl del mismo autor, véase Hernández (2008).

${ }_{14}$ Para revisar la labor de los franciscanos véase, p. ej., Domínguez Reboiras (2011).

${ }^{15}$ Consúltese la lista de las obras en Hernández (2018: 97, 99); para ver algunos detalles de ellas, véase Valero (2015). 
tarascos en la primera parte, luego una lista de unas 90 raíces verbales de tarasco, la segunda parte tiene unas 12000 entradas españolas con sus equivalencias en tarasco. En cuanto a las entradas castellanas, se basa directamente en Molina (1555) e indirectamente en Nebrija (Acero Durántez, 2003: 188). Para la historia del léxico español, contiene 23 indigenismos en las definiciones castellanas: cacles, maíz, tianguez, maguey, cacao, tamales, canoa, nahuatlato, auacate, guayaba, ocota/ ocote, ají, sabana, tamemes, petate, xicaras, mastlatl, petaca, coa, andumuqua, vanita, xacal, tomatl o expresiones que son traducciones literarias del tarasco: dormir sobre el coraçon, estar el páxaro asentado encima del ramito (Hernández, 2018: 98).

Finalmente, en 1560, fray Domingo de Santo Tomás publica en Valladolid, para la enseñanza de los futuros evangelizadores, el primer diccionario de la lengua quechua: Lexicon o Vocabulario de la lengua general del Perv, que se estima muy valioso para la etnografía o antropología, pero, desde el punto de vista del español, sigue al Vocabulario de Nebrija eliminando las entradas sin equivalencia y topónimos, antropónimos y gentilicios del Viejo Mundo. Lo interesante es que documenta el quechuismo papa. Contiene 11000 entradas: español-quechua con 4400 entradas y quechua-español con 6300 entradas. Es, además, el único diccionario bilingüe hispano-amerindio que se imprimió en España.

En 1585, en la primera imprenta fundada en Lima aparece el primer libro impreso en América del Sur: Doctrina cristiana [y catecismo para la instrucción de los indios, y de las demás personas que han de ser enseñadas en nuestra santa fe: con un confesionario y otras cosas necesarias]. Al final se halla un vocabulario de "vocablos dificvltosos de la qvichva" y más adelante el vocabulario aimara: el primer inventario léxico de esta lengua (Hernández, 2018: 104).

Un año después, en 1586, aparece el anónimo Vocabulario de la lengua castellana y de la lengua quichua ${ }^{16}$. Es un diccionario bidireccional, al parecer no se basa en su antecesor (el de fray Domingo de

16 Sobre su polémica autoría, véase Hernández (2018: 104). 
Santo Tomás) y contiene documentación de tales indigenismos como: coca o chicha.

Del siglo XVI vale la pena mencionar: Arte y dictionario de la lengua tarasca de fray Juan Bautista Bravo de Lagunas (México, 1574), Vocabulario de la lengua castellana y zapoteca de fray Juan de Córdoba (México, 1578) o Vocabulario de la lengua castellana y mixteca de los padres de la Orden de Predicadores (México, 1593).

El siglo XVII todavía cuenta con algunos manuscritos: 1642 - Vocabulario de la lengua matlaltzinga, compuesto por fraile agustino Diego de Besalenque, autor de varias obras sobre la lengua matlatzinca, 1692 -Arte de la lengua mexicana según la acostumbran hablar los indios en todo el obispado de Guadalajara del franciscano fray Juan Guerra, 1696 -Vocabulario grande de la lengua choltí (una lengua maya, hoy extinta) de fray Francisco Morán, que consta de una gramática, un confesionario y un vocabulario más algunos textos misionales y algunos otros vocabularios de las lenguas: zapoteca, otomí, tarasco o zoque (Arte breve y vocabulario de la lengua tzoque conforme se habla en el pueblo de Tecpatlán de fray Luis González, primera gramática de esta lengua de 1652), más allá de varios diccionarios mesoamericanos perdidos o conocidos de los manuscritos posteriores. Entre los perdidos se halla el que vendría a ser el primer diccionario guaraní: Vocabulario guaraní-español y español-guaraní (fecha incierta) del franciscano fray Luis de Bolaños, iniciador de las reducciones en Paraguay, el primer diccionario araucano: Diccionario en araucano, mapuche y español (1642-1643) de un anónimo araucano, Vocabulario y catecismo de las Lenguas Campa, Pira, Cuniba y Comaba, con observaciones sobre sus dialectos de Enrique Riaer, c. 1690 -un compendio sobre las lenguas amazónicas, Arte y Vocabulario de la lengua de los Caribes de Nueva Andalucía y Sermones en el mismo idioma del capuchino fray José de Carabantes (1678) o Gramáticas, Vocabularios, Catecismos y Confesionarios en diversas lenguas de Tucumán del jesuita Pedro de Añasco (c. 1600). Se trata de los materiales preparados para los misioneros sobre lenguas tonocoté, kaka, paquí o quirandí, todas del noroeste argentino. 
El siglo XVII comienza con la impresión de la primera gramática y diccionario de la lengua mapuche: Arte y gramática general enla lengva que corre en todo el Reyno de Chile del jesuita Luis de Valdivia, 1606. El vocabulario mapudungun-castellano acompaña a la gramática y contiene americanismos tales como: tambo, totora, porotos, callana 'cazuela' caracha 'sarna' ${ }^{17}$. El autor anunció la redacción de la parte castellano-mapuche, pero esta se desconoce. Otra obra del mismo jesuita es del año 1607 y es la descripción junto con un vocabulario breve (nueve páginas) de la extinta ya lengua allentiac de la Argentina central. De 1611 proviene la única obra lexicográfica de este siglo no escrita por un misionero: Vocabulario manual de las lenguas castellana y mexicana, en que se contienen las palabras, preguntas y respuestas mas communes y ordinarias, que se suelen offrecer en el trato y communicacion entre Españoles é Indios de Pedro de Arenas. El Vocabulario está divido en dos partes: la primera es castellano-mexicana y la segunda mexicano-castellana, más breve esta que aquella. Se trata de una nomenclatura con la que se pretendía facilitar la comunicación diaria en español y nahua. El total de entradas en la primera parte es de 2076, mientras que en la segunda son 1068; la obra tuvo muchísima fama y 14 reediciones aunque no se sabe si su autor tuvo la idea de la moda paralela en Europa de estas guías de conversación (como las de Berlaimont) ${ }^{18}$.

Casi simultáneamente aparece una serie de vocabularios de las lenguas andinas: Vocabulario de la lengva general de todo el Perv llamada qquichua, o del Inca de Diego González Holguín (1608), un diccionario bidireccional, considerado el más completo de los publicados en los ss. XVI-XVII: contiene más de 25000 entradas, está basado en la tradición nebrisense, pero difiere cierto carácter enciclopédico en cuanto a la frecuencia con la que la entrada es una construcción sintáctica compleja; Vocabvlario dela lengua aymara del jesuita Ludovico Bertonio (1612); Arte quechua y aymara del jesuita Diego Torres

\footnotetext{
17 Para más detalles remitimos al estudio de Ridruejo (2007b).

${ }_{18}$ Hay muy escasa información sobre la vida de este autor: véase https:// www.bvfe.es/component/mtree/autor/9234-arenas-pedro-de.html, 3.12.2020.
} 
Rubio (1603, reedición en Sevilla en 1619) y otros diccionarios suyos de aimara o quechua; Tesoro de lengva gvarani del jesuita Antonio Ruiz de Montoya, autoridad en la lengua guaraní (1639) y Arte y bocabvlario de la lengva gvarani del mismo autor (1640); y obras de lenguas minoritarias, como la del capuchino fray Francisco de Tauste de 1680: Arte y bocabulario de la lengua de los indios chaymas, cumanagotos, cores, parias -lenguas de las tribus venezolanas de la costa caribeña ${ }^{19}$.

Los diccionarios del siglo XVIII siguen las pautas de sus predecesores. Muchos de ellos se enfocan en las lenguas minoritarias, varios permanecen manuscritos y quedan dispersos en varias colecciones. Aunque cronológicamente el primero sería Arte y vocabulario del idioma huasteco de Bernardo de Quirós (1711), el que nos interesa es Vocabulario de la lengua guaraní del jesuita Blas Pretovio de 1728 cuyo único (?) ejemplar se encuentra en la Biblioteca Jaguelónica ${ }^{20}$. Czopek (2008) en su breve descripción de la obra nota que es un diccionario castellano-guaraní y que ha sido redactado por dos autores diferentes, uno de los cuales seguramente fue el jesuita (Blas Pretovio es anagrama de su verdadero nombre: Pablo Restivio) y aporta algunas informaciones acerca de la política lingüística en Paraguay y el trabajo misionero del autor. Vale la pena agregar que este diccionario,

19 Para los vocabularios manuscritos de las lenguas mayas, véase Hernández (2018: 139-155). Sobre la obra del misionero aragonés Francisco de Tauste, véase Sánchez Méndez (2012) y para los reajustes semánticos en las primeras obras lexicográficas andinas, véase Peña Torrejón (2006), entre otros.

20 Hernández (2018: 159) menciona que en el catálogo del Museo Mitre "se consigna la ficha con $\mathrm{n}^{\mathrm{o}}$ de registro 4130: Compendio de los vocablos más usados de la lengua española y guaraní, sacado del vocabulario del padre Pretovio. Curiosamente Chamorro (2018) en su trabajo sobre la vida y la obra del padre Pretovio menciona solo el Vocabulario de la lengua guaraní del año 1722, Arte de la lengua gruaraní del año 1724, Compendio de los vocablos más usados de la lengua Española y Guaraní (1729) y el Vocabulario de todos los verbos usados en la lengua guaraní (1729), todos ubicados en el Museo Mitre en Buenos Aires. 
preparado como ayuda para los co-hermanos misioneros ${ }^{21}$, es al mismo tiempo un tipo de catequismo y hasta de confesionario (Stala, 2020).

Entre otros manuscritos interesantes se hallan: Arte y vocabulario de la lengua chiquita, una lengua del oriente boliviano, del jesuita José Francisco Arce (sin fecha exacta), Vocabulario indios motilones (una tribu colombiana) de fray Francisco de Catarroja de 1738, varios vocabularios de náhuatl (c. 1760, 1778, 1788). En la colección del Museo Británico se encuentra el anónimo Diccionario de la lengua patagónica y en la Colección "Celestino Mutis" de la Biblioteca Real de Madrid"2, se hallan 19 manuscritos de vocabularios de lenguas indígenas recopilados por José Celestino Mutis que ahora alberga la Real Biblioteca de Palacio en Madrid, preparada a pedido de la Catalina II de Rusia; entre los diecinueve volúmenes se encuentran vocabularios de las lenguas: achagua, andaqui, arauaca, ceona, pariagota, mosca o motilona ${ }^{23}$.

Otras fuentes para este tipo de obras son: la Biblioteca de la Real Academia de la Historia en Madrid (allí por ejemplo se archiva el Vocabulario de los Indios de los ríos Putumayo y Caquetá de 1751) o el Archivo General de Indias en Sevilla (entre otros: Vocabulario de la isla de Pascua de 1771).

Entre los vocabularios impresos del siglo XVIII, hay que mencionar el Vocabulario quechua y de la lengua chinchaisuyo del jesuita Juan de Figueredo (1700), el Arte de la lengua moxa, una lengua de la región andina boliviana, con su vocabulario y catecismo del padre Pedro Marabán (1702), el Arte y vocabulario de la lengua lule y toconote del jesuita Antonio Machoni (1732) que se considera el léxico panquechua supradialectal, el Vocabulario Hispano-Chileno y un calepino Chileno-Hispano mas copioso del jesuita Andrés Febres (1765), el Vocabulario en lengua castellana y cora, una lengua uto-azteca, del jesuita José de Ortega (1732), el Arte de la lengua tepeguana, una

${ }^{21}$ En la p. $2 v^{0}$ el autor avisa: “Al fin pondré por aprender otros vocablos que no se me han ofrecido por aora, y pondré tambien algunos modos de hablar proprios,y eleghanmtes sacados de autores de primera clase y sea todo por amor de Dios, Salud de estas almas, y alivio de los Padres principiantes".

${ }^{22} \mathrm{http} / / /$ coleccionmutis.cubun.org/P\%C3\%A1 gina_principal, 3.12.2020.

${ }^{23}$ La lista completa en Hernández (2018: 164). 
lengua del oriente mexicano, con vocabulario del jesuita P. Benito Rinaldini (1743), el Arte del idioma maya ... y semilexicon yucateco del padre Beltrán de Santa Rosa (1746) o la Noticia de la lengua huasteca y copioso vocabulario de Carlos de Tapia Zenteno (1767).

Este ha sido un esbozo general de los primeros siglos de la lexicografía hispano-amerindia. Por supuesto, la actividad lexicográfica no solo sigue, sino aumenta con cada siglo. Es más, aparecen nuevos estudios, pero también nuevos enfoques y nuevos retos. Por no ser el tema principal de este trabajo, le dedicamos solo un pequeño párrafo aparte.

\section{La lexicografía hispanoamericana en la época moderna}

Algunas de las obras de la época colonial siguen reeditándose a lo largo del siglo XIX, pero el siglo XIX es el comienzo de proyectos de diccionarios dialectales, empezando por el Diccionario provincial casi razonado de voces y frases cubanas de Esteban Pichardo y Tapia (1836) y terminando el siglo con el Vocabulario de mexicanismos de Joaquín García Icazbalceta (1899). Los países que se suman a este tipo de proyectos son, más allá de los mencionados: Venezuela, Colombia, Perú, Chile, Ecuador, Argentina, Costa Rica, Guatemala y Honduras ${ }^{24}$.

En el s. XX siguen componiéndose diccionarios bilingües. Acero Durántez (2003) menciona a modo de ejemplo: Diccionario quiché-español de J. de León (Guatemala, 1955), Diccionario español-aymara, aymara-español de Louisa R. Stark y Pieter C. Muysken (Lima, 1988), Diccionario de la lengua phorhépecha: español-phorhépecha, phorhépecha-español (México, 1978). Pero la lexicografía moderna abarca no solo los diccionarios bilingües, sino todo tipo de obras dedicadas a las lenguas regionales. Como en 1996 observó Luis Fernando Lara:

La conquista de América no solamente produjo el fenómeno objetivo de la expansión del español por una gran parte del nuevo continente, tal como lo intuyó Nebrija, sino que estableció un hecho simbólico cuya vigencia permanece en nuestra concepción social de la lengua española: creó un

24 La lista completa de las obras se halla, p. ej., en Barcia (2006: 19-20). 
sujeto histórico y una localización geográfica de nuestra lengua de acuerdo con los cuales España, la metrópoli, y su corte o sus autores literarios determinan la "verdadera imagen de la lengua española" — según expresión reciente de un lexicógrafo español—, mientras que Hispanoamérica se convirtió en permanente periferia de la lengua y objeto pasivo de los juicios de corrección peninsulares: reiteradamente sospechosa de barbarismo, aunque muchas veces perdonada indulgentemente por lo pintoresco de sus amerindianismos, sólo se la puede mirar como arcaica, como andalucista, como objeto de una siempre posible redención (Lara, 1996: 345),

por lo que hoy en día se discute no solo qué léxico elegir y cómo documentarlo, sino también el lugar que ocupa el vocabulario americano en todo el espectro del léxico panhispano. De hecho, en la edición de 1992 del diccionario de la Academia, aparece por primera vez la marca de uso españolismo (Stork, 2008: 595) o sea que los regionalismos españoles empiezan a igualar su posición "exótica" con los regionalismos hispanoamericanos.

En la literatura actual muchas veces se diferencia entre los diccionarios generales y regionales (lo que aproximadamente equivaldría a la diferencia entre integrales y diferenciales según la propuesta de Haensch): los generales hasta ahora se han escrito exclusivamente en España y abarcan la totalidad de la lengua, mientras que los regionales aparecen en cualquier territorio hispanohablante (Andalucía, Colombia, Yucatán, etc.) y se ven como complementarios. Lara (1996: 355) observa que muchas veces los diccionarios de regionalismos adquieren un valor ideológico y reivindicativo de la comunidad y cita a modo de ejemplo el Diccionario de aztequismos. De hecho, Javier Pérez (2003) subraya esta combinación colonial de purismo conceptual y regionalismo léxico en las principales obras de tipo regional del s. XIX: el ya citado Diccionario provincial casi razonado de voces y frases cubanas de Esteban Pichardo (1836), el Diccionario Indo-Hispánico o venezolano español de Miguel Carmona (1858-1859), las Apuntaciones sobre el lenguaje bogotano de Rufino José Cuervo (1867-1872), el Diccionario de peruanismos de Juan de Arona (1871), los Hondureñismos de Alberto Membreño (1898) por citar solo algunos y hasta llegar al 
s. XX: el Diccionario de chilenismos y de otras voces y locuciones viciosas de Manuel A. Román (1901-1918), los Argentinismos. Neologismos y barbarismos de Lisandro Segovia (1911) o las Voces chilenas y chilenismos de José Toribio Medina (1925).

El siglo XX cuenta con varios diccionarios de los "-ismos" (se citan varios ejemplos en el artículo de Javier Pérez, 2003: 259-261) y se siguen editando obras nuevas en el s. XXI (p. ej. Diccionario de habla de los argentinos, 2008, 2a ed., Buenos Aires: Emecé Ediciones o Diccionario de uso del español en Chile, 2010, Academia Chilena de Lengua).

En 1912 el lexicógrafo español Miguel de Toro Gisbert publica su tratado Americanismos y, al distinguir tres tipos de léxico: los diccionarios de vicios (galicismos y barbarismos), los diccionarios de indigenismos y los que reúnen voces de cada país americano, empieza la discusión sobre el término americanismo y estimula la redacción de varios trabajos teóricos y prácticos, esto es, los diccionarios. Entre ellos destacan el de 2010: Diccionario de americanismos, publicado por la Asociación de Academias de la Lengua Española ${ }^{25}$ o el no concluido aún Diccionario de americanismos, elaborado bajo la dirección de César Alonso Hernández ${ }^{26}$.

Últimamente entre los proyectos más importantes se menciona el de Luis Fernando Lara Diccionario del español de México, recopilación del léxico mexicano en su dimensión "integral", editado en $2010^{27}$, al que acompaña el Diccionario integral del español de la Argentina de Plager (2008), pero aparecen también proyectos "diferenciales" que recopilan el léxico que no es común en otros países. Así, desde 1976 se viene elaborando un proyecto denominado Nuevo diccionario de americanismos, dirigido por Günter Haensch y Reinhold

${ }^{25}$ Descripción en: http://www.rae.es/obras-academicas/diccionarios/ diccionario-de-americanismos.

${ }_{26}$ Para la descripción del proyecto, véase, p. ej. https://elpais.com/diario/2005/10/26/cultura/1130277604_850215.html, 15.10.2019.

${ }^{27}$ El diccionario accesible en línea: http://www.cervantesvirtual.com/obravisor/diccionario-del-espanol-usual-en-mexico--0/html/, 3.10.2020, la reseña del diccionario en Hernández Cruz (2015). 
Werner $^{28}$, que consiste en la elaboración de un diccionario para cada uno de los 19 países hispanoamericanos (hasta ahora han salido los de colombianismos, argentinismos, uruguayismos y cubanismos).

Como calcula Rodríguez González (1995), hace ya más de dos décadas "en los últimos siglos son más de dos mil publicaciones, entre diccionarios y glosarios, que se han ocupado de recopilar y definir las voces y expresiones típicas de cada país hispanoamericano".

\section{La metalexicografía hispanoamericana}

Con el paso del tiempo aparecen no solo las obras nuevas, sino también la distinción entre la lexicografía en sentido del arte de componer diccionarios y la metalexicografía que comprende la teoría, crítica e historia de los diccionarios. Así, se dice que la primera muestra lexicográfica es la obra de Pedro Mártir de Anglería, cortesano al servicio de los Reyes Católicos, quien en su obra epistolar en forma de ocho libros titulada Décadas del Nuevo Mundo (1516-1530) describe su época basándose en las informaciones obtenidas de entrevistas con los navegantes y conquistadores. Sobre todo las primeras tres décadas, publicadas en Alcalá en 1516, son importantes desde el punto de vista lexicográfico porque al final de la tercera se encuentra un glosario denominado Vocabula Barbara con una lista de indigenismos:

Las voces indígenas, curiosamente latinizadas, que él emplea en sus Décadas, las escuchó, probablemente hispanizadas, de sus viajeros informantes que llegaban de las Indias o las leyó manuscritas en las narraciones y cartas (en las que seguramente se empleaba la lengua española) que sin duda consultó

dice Moreno de Alba (1996: 4), quien a su vez examina los nombres propios de esta lista. En 1627 fray Pedro Simón redacta Tabla para inteligencia de algunos vocablos desta historia, anexo para sus Noticias

28 La descripción del proyecto se halla en Haensch y Werner (1978), la descripción bastante detallada del Diccionario de los argentinismos en Rodríguez González (1995), la reseña del diccionario de los cubanismos y argentinismos en Valadez (2002), por citar solo algunas publicaciones al respecto. 
Historiales, otra "pieza fundadora de la reflexión lexicográfica", como los llama Javier Pérez (2003: 252).

Como principio de la sistemática lexicografía hispanoamericana se suele aceptar el Diccionario geográfico-histórico de las Indias Occidentales o América de Antonio de Alcedo (1786-89) que en el quinto volumen incluye como anexo el repertorio de las voces americanas ${ }^{29}$, aunque habrá que esperar hasta 1846 cuando Andrés Bello publica su gramática destinada a los hispanohablantes americanos. Como dice Martínez (1968), en este lapso de tiempo predomina el léxico sobre la gramática.

En cuanto a la crítica de los diccionarios, los primeros ensayos de este tipo aparecen en el s. XIX (Javier Pérez, 2003: 263-265). Entre los fundadores de esta corriente se halla indudablemente Andrés Be1lo, al que siguen varios críticos que publican las reseñas de varios diccionarios (p. ej. José Martí "Muestra de un ensayo de diccionario de vocablos indígenas" (1881), Hugo Schuchardt "Apuntaciones para la crítica sobre el lenguaje maracaibero" (1883) o varias reseñas del cubano Juan Miguel Dihigo (1917-1929)). Los ejemplos de las reseñas modernas se citan a lo largo de este trabajo.

Si se trata de la historia de la lexicografía, obras de esta índole comprenden historias generales de la lexicografía hispanoamericana, historias regionales, historias especiales, biografías y estudios de autor y bibliografías. Javier Pérez (2003: 265-272) destaca a los fundadores de este tipo de repertorios, a saber: Juan de Arona en su Diccionario de peruanismos (1871) adjunta una lista de obras y autores que lo han precedido y la titula "Bibliografía de americanismos"; Rodolfo Lenz (y su "Bibliografía crítica de las obras sobre americanismos" como introducción a su Diccionario etimológico de las voces chilenas derivadas de lenguas indígenas americanas (1905-1910)) y Miguel de Toro y Gisbert (quien concibe el texto "Los diccionarios de americanismos" para sus ensayos titulados Americanismos (1912)). Entre los trabajos modernos se hallan, entre otros: el artículo de Martínez (1968), citado

29 Sobre los vocablos alusivos a Nueva España en esta obra, véase Kamenetskaia (2018). 
a lo largo de este texto, varios trabajos de los citados en estas páginas Günther Haensch y Reinhold Werner, las investigaciones de López Morales (p. ej. "Diccionarios generales de americanismos" de 1999), de Manuel Alvar Ezquerra "Aportaciones recientes a la lexicografía del español de América" (1989) o de Claudio Chuchuy "Los adjetivos en los diccionarios contrastivos del español de América” (1994), pero también trabajos como "Panorama de lexicografía boliviana" (1988), "El español de Cuba: Situación bibliográfica" (1971), La lengua de Martín Fierro (1925), o La lengua de Bolívar (1961). Más obras de este tipo se hallan en la bibliografía de Fabbri (1979), el artículo de Martínez (1968) o en el de Javier Pérez (2003).

Por supuesto es solo una aproximación a la vasta área de la lexicografía hispanoamericana. Ya en 1968 Martínez (1968: 92) enumera toda una lista de problemas de la lexicografía americana: americanismos, provincialismos, regionalismos, indigenismos, topónimos, europeísmos o léxico de autores individuales. Hay proyectos nunca concluidos, como el de Pedro Henríquez Ureña: Diccionario histórico de indigenismos ${ }^{30}$. Es más, se encuentran y editan obras hasta hace poco desconocidas, como el primer diccionario de argentinismos, elaborado entre 1875-1879 y publicado en 2006 (Barcia, 2006) u obras dedicadas a las lenguas de tribus muy reducidas, como el Diccionario de la lengua ette, una lengua del norte colombiano (Niño Vargas, 2018).

Entre los retos actuales se mencionan desde las empresas de tipo más usual, como buenos diccionarios escolares o diccionarios de español de un país concreto como segunda lengua (Chávez Fajardo, 2008), hasta el cambio de óptica en la confección de las obras lexicográficas como, por ejemplo, la propuesta de Stork (2008) quien, partiendo desde el punto de vista de la ecolingüística, aboga por la interpretación pluricentrista de la lengua y como ejemplo de este tipo de obras enumera el Diccionario panhispánico de dudas (2005) - el primer diccionario conjunto de las 22 Academias de la Lengua de España e Hispanoamérica, el Diccionario del español usual en México (1996),

30 Para este aspecto de la obra de Henríquez Ureña y el mismo proyecto, véase Vaquero Ramírez (1985). 
dirigido por Lara o el Diccionario de uso del español de América y España de Lucena Cayuela (2002). Como respuesta a esta demanda, aunque sea parcial, en 2003 se publicó en México un diccionario escolar: Dime. Diccionario inicial del español de México de Raúl Ávila.

Sumando las propuestas más recientes a las lagunas e incógnitas que encontramos a cada paso al investigar las obras históricas, no cabe duda de que la lexicografía hispanoamericana presenta un amplísimo campo de búsquedas e investigaciones.

\section{Bibliografía}

ACERO DURÁNTEZ, I. (2003), "La lexicografía plurilingüe del español", en: Medina Guerra, A. M. (ed.), Lexicografia española, Ariel Lingüística, Barcelona, pp. 175-204, https://doi.org/10.12795/PH.2008.v22.i01.01.

AHUMADA, I. (dir. y ed.) (2006-2014), Diccionario bibliográfico de la metalexicografia del español, Publicaciones de la Universidad de Jaén, Jaén. ALVAR EZQUERRA, M. (2003), De antiguos y nuevos diccionarios del español, Arco/Libros, Madrid.

AZORÍN FERNÁNDEZ, D. (2004), Los diccionarios del español en su perspectiva histórica, Universidad de Alicante, Alicante.

BARAN, M., STALA, E. (2019), "El Vocabulario trilingüe (c. 1550): la herramienta moderna en servicio del pasado", en: Santos Rovira, J. M. (ed.), Raices y horizontes del español: perspectivas dialectales, históricas y sociolingüísticas, Editorial Axac, Lugo, pp. 99-107.

BARCIA, P. L. (2004), Los diccionarios del español de la Argentina, Academia Argentina de Letras, Buenos Aires.

BARCIA, P. L. (2006), Un inédito Diccionario de argentinismos del siglo $X I X$, Academia Argentina de Letras, Buenos Aires.

BOLLES, D. (2003), The Mayan Franciscan Vocabularies: A Preliminary Survey, [on-line] http://www.famsi.org, 20.02.2019.

CENSABELLA, M. (1999), Las lenguas indigenas de la Argentina, Eudeba, Buenos Aires. 
CHAMORRO, G. (2018), “El artista de la lengua guaraní: vida y obra del misionero Paulo Restivo", [on-line] https://www.berose.fr/article1483. html?lang=fr, 7.02.2019.

CHÁVEZ FAJARDO, S. (2008), "La lexicografía en Chile: hiatos por descubrir", en: Azorín Fernández, D. et al. (ed.), El diccionario como puente entre las lenguas y culturas del mundo. Actas del II Congreso Internacional de Lexicografía Hispánica, Alicante, pp. 517-521.

CONDE SILVESTRE, J. C. (2007), Sociolingüistica histórica, Gredos, Madrid.

CZOPEK, N. (2008), "The Guarani Language in the Manuscript of Berlin Collection of the Jagiellonian Library”, Fibula, 1, Kraków, pp. 25-29.

DOMÍNGUEZ REBOIRAS, S. (2011), "Los franciscanos de Nueva España y las lenguas indígenas", en: Oesterreicher, W., Schmidt-Riese, R. (eds.), Esplendores y miserias de la evangelización de América. Antecedentes europeos y alteridad indígena, Walter de Gruyter, Berlin-New York, pp. 301-330.

FABBRI, M. (1979), A Bibliography of Hispanic Dictionaries. Catalan, Galician, Spanish [...], Galeati, Imola, https://doi.org/10.1075/lplp.7.3.10woo.

GARCÍA AGUILAR, M. I., RUEDA RAMÍREZ, P. J. (comp.) (2010), Leer en tiempos de la Colonia: imprenta, bibliotecas y lectores en la Nueva España, UNAM, Centro Universitario de Investigaciones Bibliotecológicas, México, [on-line] http://ru.iibi.unam.mx/jspui/bitstream/IIBI_UNAM/ L33/1/leer_tiempos_colonia.pdf, 20.1.2019.

GILI GAYA, S. (1947), Tesoro lexicográfico (1492-1726), (A-CH), Consejo Superior de Investigaciones Científicas, Madrid, https://doi.org/10.22201/ cuib.9786070207501e.2010.

GRUDA, S. (2018), Language and Culture Contact Phenomena in the Ayer Vocabulario Trilingüe in Spanish, Latin and Nahuatl, Wydział “Artes Liberales", Uniwersytet Warszawski, Warszawa.

GUIBOVICH PÉREZ, P. (2019), Imprimir en Lima durante la colonia: historia y documentos, 1584-1750, Vervuert Iberoamericana, Madrid, https:// doi.org/10.31819/9783964568625.

HAENSCH, G., WERNER, R. (1978), “Un Nuevo Diccionario de americanismos: proyecto de la Universidad de Augsburgo", Boletín del Instituto Caro y Cuervo, 23 (1), pp. 1-40. 
HAUSMAN, F. J. (1991), Wörterbücher Dictionaries. Dictionnairies: ein internationales Handbuch zur Lexicographie, Walter de Gruyter, Berlin. HERNÁNDEZ, E. (2006), "Influencias de método y concepción entre los vocabularios novohispanos del siglo XVI”, en: Pérez Pascual, J. I., Córdoba Rodríguez, F., Campos Souto, M. (coords.), América y el diccionario, Anexos de Revista de Lexicografía, Universidad da Coruña, A Coruña, pp. 63-78.

HERNÁNDEZ, E. (2008), "El Arte de la lengua mexicana y castellana de Fray Alonso de Molina: morfología y composición”, Estudios de Cultura Náhuatl, 39, México, pp. 167-206.

HERNÁNDEZ, E. (2018), Lexicografía hispano-amerindia 1550-1800, Vervuert Iberoamericana, Frankfurt, https://doi.org/10.31819/978395 4877591.

HERNÁNDEZ CRUZ, A. (2015), "Luis Fernando Lara (dir.), Diccionario del español de México. El Colegio de México, México, 2010; 2 ts.: 1706 pp.” (reseña), Nueva Revista de Filología Hispánica, LXIII, nº 2, México, pp. 473-477.

JAVIER PÉREZ, F. (2003), "La metalexicografía en Hispanoamérica”, Lingüística española actual, 23 (1-2), Madrid, pp. 249-271.

KAMENETSKAIA, S. (2018), "Léxico novohispano en el Vocabulario de Antonio de Alcedo", Nueva Revista de Filología Hispánica, 66 (2), México, pp. 627-649, https://doi.org/10.24201/nrfh.v66i2.3428.

KARTTUNEN, F. (1995), "The Roots of Sixteenth-Century Mesoamerican Lexicography”, en: Kachru, B., Kahane, H. (eds.), Cultures, Ideologies and the Dictionary. Studies in Honor of Ladislav Zgusta, Max Niemeyer, Tübingen, pp. 75-88, https://doi.org/10.1515/9783110957075.75.

LARA, L. F. (1996) "Por una redefinición de la lexicografía hispánica", Nueva Revista de Filología Hispánica, 64 (2), México, pp. 345-364, https:// doi.org/10.24201/nrfh.v44i2.1942.

LÓPEZ MORALES, H. (2000), "Diccionarios generales de americanismos", en: Ahumada, I. (ed.), Cinco siglos de lexicografía española. IV Seminario de Lexicografía Hispánica, Universidad de Jaén, Jaén, pp. 161-176. MOLINA, ALONSO DE (1555), Vocabulario de la lengua castellana y mexicana, Juan de Pablos, México. 
MORENO DE ALBA, J. G. (1996), “Indigenismos en las Décadas del Nuevo Mundo de Pedro Mártir de Anglería”, Nueva Revista de Filología Hispánica, 64 (1), México, pp. 1-26.

MARTÍNEZ, F. A. (1968), "Lexicography”, en: Lado, R., Mcquown, N. A., Saports, S, Sebeok, T. (eds.), Current Trends in Linguistics. 4. Iberoamerican and Caribbean Linguistics, Mouton, The Hague, pp. 84-105.

MEDINA GUERRA, A. M. a (2003), Lexicografía española, Ariel Lingüística, Barcelona.

MENÉNDEZ PIDAL, R. (1944), "Los incunables americanos. Prólogo a la Colección de Incunables Americanos”, Cultura Hispánica, Madrid, pp. V-XXII.

MUÑOZ Y MANZANO, C. (1892), Bibliografía española de lenguas indígenas de América, Est. Tipográfico "Sucesores de Rivadeneyra”, Madrid. NIEDEREHE, H. J. (1994-2015), Bibliografía cronológica de la lingüística, la gramática y la lexicografía del español, t. I-V, John Benjamins Publishing Company, Amsterdam.

NIÑO VARGAS, J. C. (2018), Diccionario de la lengua ette, Universidad de los Andes, Instituto Colombiano de Antropología e Historia, Bogotá, http://dx.doi.org/10.30778/2017.16.

PEÑA TORREJÓN, J. G. (2006), “La primera lexicografía andina: paralelismos, préstamos y reajustes semánticos en quechua y aimara (15321640)", en: Pérez Pascual, J. I., Córdoba Rodríguez, F., Campos Souto, M. (coords.), América y el diccionario, Anexos de Revista de Lexicografía, Universidad da Coruña, A Coruña, pp. 113-124.

PLAGER, F. (coord.) (2008), Diccionario integral del español de la Argentina, Voz Activa, Buenos Aires.

RIDRUEJO, E. (2007), El Arte de la lengua de Chile de Luis de Valdivia, Ministerio de Asuntos Exteriores y Cooperación. Agencia Española de Cooperación Internacional, Madrid.

RODRÍGUEZ GONZÁLEZ, F. (1995), “Apuntes sobre la lexicografía hispanoamericana”, Revista Española de Lingüística, 25 (1), Madrid, pp. 169-180.

ROMERO RANGEL, L. (2006), "El quehacer lexicográfico en los Vocabularios de Alonso de Molina”, en: Girón Alconchel, J. L., Bustos Tovar, J. J. 
(eds.), Actas del VI Congreso Internacional de Historia de la Lengua española, II, Arco Libros, Madrid, pp. 1616-1621.

SÁNCHEZ MÉNDEZ, J. P. (2012), “Arte y bocabvlario de la lengva de los indios chaymas...: una empresa lingüística del aragonés fray Francisco de Tauste”, Archivo de Filología Aragonesa (AFA), 68, Zaragoza, pp. 123-142.

STORK, Y. (2008), "La situación lexicográfica del español hispanoamericano. Consideraciones desde la perspectiva de la ecolingüística", en: Azorín Fernández, D. et al. (ed.), El diccionario como puente entre las lenguas y culturas del mundo. Actas del II Congreso Internacional de Lexicografía Hispánica, Alicante, pp. 594-600.

VALADEZ, C. D. (2002), "Günther Haensch (...)” (reseña), Nueva Revista de Filología Hispánica, 50 (1), México, pp. 238-245.

VALERO, P. (2015), "La terminología cristiana en el Vocabulario y el Arte de la lengua del fraile franciscano Maturino Gilberti. El escrito misionero como mediación intercultural de carácter multidisciplinar”, Mutatis $\mathrm{Mu}$ tandis, 8 (1), Santiago de Chile, pp. 215-238.

VAQUERO RAMÍREZ, M. T. (1985), "Pedro Henríquez Ureña y la lingüística indigenista", [on-line] http://smjegupr.net/wp-content/ uploads/2015/03/4-Pedro-Henriquez-Ure--a-y-la-lingueistica-indigienistapor-Mar--a-T.-Vaquero-de-Ram--rez.pdf, 18.01.2019.

ZALDÍVAR, J. I., "Fray Andrés de Olmos (1485-1571): de Oña a la Huasteca mexicana", [on-line] http://www.onienses.com/PDFS/Andresdeolmos. pdf, 22.01.2019.

ZWARTJES, O., HOVDHAUGEN, E. (2004-2014), Missionary Linguistics/ Lingüística Misionera, John Benjamins Publishing Company, Amsterdam.

\section{Enlaces de Internet}

http://coleccionmutis.cubun.org/P\%C3\%A1 gina_principal, 22.02.2019.

https://www.bvfe.es/component/mtree/autor/9234-arenas-pedro-de.html, 22.02.2019.

http://www.cervantesvirtual.com/obra-visor/diccionario-del-espanol-usualen-mexico--0/html/, 2.02.2019. 
http://www.cervantesvirtual.com/obra-visor/universal-vocabulario-en-latiny-en-romance-tomo-ii--0/html/003fa87a-82b2-11 df-acc7-002185 ce6064_509.html, 28.02.2019.

http://www.rae.es/obras-academicas/diccionarios/diccionario-de-americanismos, 26.02.2019.

https://elpais.com/diario/2005/10/26/cultura/1130277604_850215.html, 26.02.2019. 